\title{
MEDICALIZATION OF SEXUAL DESIRE
}

\author{
Jacob Stegenga ${ }^{1}$ \\ ${ }^{1}$ University of Cambridge \\ Original scientific article - Received: 21/05/2021 Accepted: 08/11/2021
}

\begin{abstract}
Medicalisation is a social phenomenon in which conditions that were once under legal, religious, personal or other jurisdictions are brought into the domain of medical authority. Low sexual desire in females has been medicalised, pathologised as a disease, and intervened upon with a range of pharmaceuticals. There are two polarised positions on the medicalisation of low female sexual desire: I call these the mainstream view and the critical view. I assess the central arguments for both positions. Dividing the two positions are opposing models of the aetiology of low female sexual desire. I conclude by suggesting that the balance of arguments supports a modest defence of the critical view regarding the medicalisation of low female sexual desire.
\end{abstract}

Keywords: medicalization; female sexual interest/arousal disorder; philosophy of medicine; disease; controversial diseases; philosophy of psychiatry

\section{Introduction}

Medicalisation is a social phenomenon in which conditions that were once under legal, religious, personal or other jurisdictions are brought into the domain of medical authority. Low sexual desire in females has been medicalised, pathologised as a disease, and intervened upon with a range of pharmaceuticals. There are two polarised positions on the medicalisation of low female sexual desire. The mainstream viewimplicitly held or explicitly articulated by many physicians, patient advocacy groups, pharmaceutical companies, activists, and policy makers - is that the medicalisation of low female sex desire is appropriate. Many females with low sexual desire suffer distress, on the mainstream 
view, and medicine is the correct jurisdiction for the alleviation of such suffering. Sexual desire, on this view, is like an appetite-a function of biological features such as hormone balances or neurotransmitter concentrations - and low sexual desire can be modulated by exogenous interventions on these biological features.

The critical view-implicitly held or explicitly articulated by some psychiatrists, psychologists, journalists, activists, and academic commentators - is that the medicalisation of low female sexual desire is pernicious. These critics argue that low sexual desire ought to be understood not as a disease but rather as a phenomenon arising out of a particular social context, and thus medicine is not the correct jurisdiction for females who experience low sexual desire. Sexual desire, on the critical view, is not solely or typically a function of biological causes but rather is typically a function of social causes - perhaps as a result of stress or fatigue or uneducated partners or toxic relationships or other diseases or even as a harmful effect of medications for those other diseases. Such critics sometimes claim that the very notion that one's sexual desires are dysfunctionally low involves appealing to culturally-determined norms of sexuality, or relational imbalances between the sexual desires of a female and her partner, and are not necessarily intrinsic harms to a female with low desire herself.

In short, there exist two antagonist positions regarding the medicalisation of low female sexual desire. In practice the positions are not always so clearly demarcated - the psychiatrist Rosemary Basson, for example, contributed to the development of the contemporary diagnostic category of low female sexual desire while also criticising the use of pharmaceutical interventions for the alleged disease. Nevertheless, there are clear trenches on the ground, and both sides are armed with statistics, science, patient testimonies, campaigns, and principled arguments of varying quality.

When asked about the potentially nefarious consequences of medicalising low female sexual, Irwin Goldstein, a urologist and prominent defender of the medicalisation of female sexual desire, deflected the concern by responding "that's a question for some philosopher" (Quoted in Moynihan 2003). Here I describe and assess several of the most important arguments from both positions regarding the medicalisation of low female sexual desire. ${ }^{1}$ I begin by tracing conceptualisations of low female sexual desire beginning in the early twentieth century (\$2). This is stage-setting. I

\footnotetext{
${ }^{1}$ In this paper I use the term 'female'; although the scientific literature that this paper addresses often uses the terms 'woman' and 'female' interchangeably, the putative disease in question targets the biological category 'female' (and this term appears in the name of the disease), and an inclusion criterion for the clinical studies is status as a biological female.
} 
proceed to articulate and assess several of the most important arguments for the mainstream view $(\$ 3)$ and the critical view $(\S 4)$. Dividing the two positions are opposing models of the aetiology of low female sexual desire (§5). I conclude by suggesting that the balance of arguments supports a modest defence of the critical view regarding the medicalisation of low female sexual desire $(\S 6)$.

\section{Conceptualizations of Low Female Sexual Desire}

Though Foucault flagged the middle of the nineteenth century as the moment in which a sub-discipline of medicine devoted to sex appeared, the focus during this nascent period of sex medicine was the 'paraphilias' or 'sexual perversions' (sexual desire for an atypical object or activity in which such desire causes distress to the desirer or harm to others). ${ }^{2}$ Low sexual desire in females has been pathologized by psychiatry and related disciplines since the final years of the nineteenth century (Angel 2010). Marital advice manuals, psychoanalytic texts, psychiatric diagnostic manuals, sexologists, and feminist critics of much of this discourse have articulated numerous theories about low female sexual desire, including what constitutes female sexual dysfunction, and its causes and optimal modes of treatment. There are two broad classes of models of low female sexual desire: an appetitive or biological model, which holds that low female sexual desire is a result of a dysfunction in a physiological capacity, and a social or contextual model, which holds that low female sexual desire is a result of features of a female's social or cultural context (\$5).

The way in which low female sexual desire has been conceived has changed often, as illustrated by the various editions of the DSM. The first edition, published in 1952, included 'frigidity', which was the closest of the female sexual dysfunctions in this edition to what we would now call low sexual desire-frigidity was characterised as disinterest in heterosexual intercourse or lack of pleasure from intercourse (other female sexual dysfunctions in the first edition included 'involutional melancholia', dyspareunia, and 'nymphomania'). After the sexual revolution of the 1960s and 1970s, the diagnosis of too much desire (nymphomania) was eliminated from the third edition, published in 1980. The third edition added the category 'inhibited sexual desire' as the diagnosis for low sexual desire in both males and females. The revision to

\footnotetext{
${ }^{2}$ The Russian physician Heinrich Kaan published his 'Psychopathia Sexualis' in 1846, in which he reinterpreted Christian sins into medical diseases; he characterised masturbation and fantasies to be the basis sexual disorders. In Foucault's 1974-75 lectures at College de France he noted that Kaan's book "was the first treatise of psychiatry to speak only of sexual pathology but the last to speak of sexuality solely in Latin". Kraft-Ebbing's more influential book of the same title appeared forty years later.
} 
the third edition, published in 1987, perhaps cleansing itself of its psychoanalytic hangover, renamed inhibited sexual desire as 'hypoactive sexual desire disorder' (again for both males and females). The present edition of the DSM is the fifth, published in 2013. Hypoactive sexual desire disorder has been divided into a male version (male hypoactive sexual desire disorder), and a female version: female sexual interest/arousal disorder.

Parallel to the evolution of the DSM, developments in the scientific and feminist study of sex provided new ways of conceiving of disorders of sexual desire. From Freud's psychoanalysis and Kinsey's statistics, from the laboratory work of Masters and Johnson, from feminist-inspired sociological, psychological and psychiatric work of those such as Hite and Tiefer and Basson, we now have multiple conceptualisations of the causes and constituents of low female sexual desire.

Freud developed psychoanalysis in part based on the idea that many of our psychopathologies are based on forms of psychological repression, and he most prominently applied this to sex. The frigidity of some women, according to Freud, was a result of psychogenic causes. Famously, Freud (1905) claimed that clitoral orgasms are a sign of immature sexual development, which held some sway into the middle of the twentieth century. Kinsey was critical of the psychoanalytic approach to sexual desire, and instead adopted a 'capacity' model, which held that different people had differing intrinsic sexual capacities. These capacities were physiological in nature, and they manifest in behaviour, specifically the frequency of a person's sexual activities. Females, on average, had lower sexual capacities than males, claimed Kinsey. Kinsey thought that such variability in a physiological sex capacity better explained variability in sexual desires compared with a repression model. ${ }^{3}$ Thus Kinsey foreshadowed a disease model of low sexual desire.

This approach was continued by the laboratory studies of Masters and Johnson. They observed people having sexual intercourse and masturbating, and ultimately recorded over ten thousand orgasms while measuring various physiological features, which formed the empirical basis of their four-phase 'sexual response cycle': excitement, plateau, orgasm, and resolution. This theory was influential; for example, it was

\footnotetext{
${ }^{3}$ Kinsey wrote: "There is an inclination among psychiatrists to consider all unresponding individuals as inhibited, and there is a certain skepticism in the profession of the existence of people who are basically low in capacity to respond. This amounts to asserting that all people are more or less equal in their sexual endowments, and ignores the existence of individual variation. No one who knows how remarkably different individuals may be in morphology, in physiologic reactions, and in other psychologic capacities, could conceive of erotic capacities (of all things) that were basically uniform throughout a population" (Cited in Irvine 1990, 36). See also Weinrich (2014).
} 
adopted and modified by psychologists and psychiatrists revising the DSM. A central concern of the work of Masters and Johnson was to develop therapies for sexual dysfunctions, including physical problems such as vaginismus (spasms of the pelvic muscles which makes intercourse painful or impossible). Although the sexual response cycle was characterised in strictly physiological terms, Masters thought that sexual dysfunctions were usually due to psychogenic causes. ${ }^{4}$

Critics argued that the human sexual response cycle theorised by Masters and Johnson is less apt for females than it is for males (see Basson 2000; Wood, Koch, and Mansfield 2006; Meana 2010). Their model did not include desire, assuming that desire occurred spontaneously. Though it was dubbed a 'cycle', critics called it 'linear', because it began with arousal and ended with orgasm and resolution. Critics noted that it ignored quality of relationships or other features of a female's social context that can influence sexual experience. More recent theories of female sexual response have attempted to accommodate these considerations. Basson, for example, has argued that female sexual desire is typically responsive (to cues, partner initiation, arousal) rather than spontaneous; that female sexual experience is typically 'circular', in which arousal can lead to desire and satisfaction can generate new desire; and that female sexual desire is modulated by social contexts such as relationship intimacy (see Basson 2000; Meana 2010).

By the late 1970s, the most common form of female sexual dysfunction, the general term for the cluster of diseases of which low female sexual desire is one, was no longer physical problems like vaginismus, but rather involved low sexual desire (Irvine 1990; Kleinplatz 2018). This was the problem that sex therapists were most often seeing in their practice (See Irvine 1990; Everard et al. 2000; and the references in Meana 2010). The disease category for low female sexual desire today is 'female sexual interest/arousal disorder'. To be diagnosed with this disease, four conditions must be met: a female must have at least three of the defining symptoms, the symptoms must persist for at least six months, those symptoms must cause her distress, and the symptoms should not be better explained by other medical conditions or relationship problems or medications. The defining symptoms are an absence of, or reduction in:

- interest in sexual activity,

- sexual thoughts or fantasies,

- initiation of sexual activity and reception of a partner's initiatives,

\footnotetext{
${ }^{4}$ Their first book was Masters and Johnson (1966). See also Fishman (2007).
} 
- excitement or pleasure during sexual activity in most sexual encounters,

- interest and arousal in response to sexual cues,

- genital or non-genital sensations during sexual activity in most encounters. (DSM-5; see also Brotto 2010).

This alleged disease, along with its predecessor (hypoactive sexual desire disorder), is the focal point for the debate regarding the medicalisation of low female sexual desire.

\section{The Mainstream View}

The mainstream view regarding the medicalisation of low female sexual desire is that this condition is a genuine disease, and thus it ought to be in the domain of medicine and is an apt target for diagnosis and medical intervention. Sexual functioning is a bodily phenomenon, on the mainstream view, and thus sexual dysfunctions are diseases like other bodily dysfunctions. Low sexual desire can cause various forms of suffering. Since medicine can sometimes help alleviate some forms of suffering, at least when such suffering is caused by a disease, there is a principled reason to think that low female sexual desire should be in the jurisdiction of medicine.

The mainstream view has a wide range of adherents. As we saw in $§ 2$, the American Psychiatric Association has codified the condition as a disease in various editions of the DSM. Prominent medical scientists such as Irwin Goldstein and the sisters Laura Berman and Jennifer Berman have for decades promoted low female sexual desire as a disease to be treated with pharmaceuticals. Millions of prescriptions have been written in the United States for off-label testosterone use for low female sexual desire, and two drugs have been approved by the FDA for the condition (flibanserin and bremelanotide), though both have extremely modest beneficial effects and a range of harms (discussed below) ${ }^{5}$ In a survey of nearly two thousand professionals attending four medical conferences, $85 \%$ believed that hypoactive sexual desire disorder is a genuine medical problem (Bachman 2006). ${ }^{6}$ We saw above that a spectrum of scholars have held low female sexual desire to be a disease, from Freud and Kinsey and Masters to Brotto and Basson.

\footnotetext{
${ }^{5}$ Regarding off-label testosterone prescriptions, see Simes and Snabes (2011)

${ }^{6}$ These were conferences of the American College of Obstetricians and Gynecologists, the Endocrine Society, the North American Menopause Society, and the American Society for Reproductive Medicine.
} 
The primary arguments for the mainstream view are:

The Argument from Suffering

The Appetitive Argument

The Argument from Female Equality

The Argument from Treatment Success

I will assess these arguments in that order.

\subsection{The Argument from Suffering}

The Argument from Suffering notes the prevalence of females with low sexual desire who experience distress from their condition. This argument is often buttressed by appealing to survey data which suggests that a very large percentage of females experience one or more of the symptoms that constitute the definition of the disease category. One particularly controversial report claimed that $43 \%$ of women suffer from some sort of sexual dysfunction (Laumann, Paik, and Rosen 1999; Berman, Berman, and Goldstein 1999; see Moynihan 2003 for criticism of this statistic). Critics claim that this figure is grossly exaggerated. Nevertheless, the most common problem that motivates visits to sex therapists for females is low sexual desire (see Irvine 1990; Kleinplatz 2018). Sometimes the widespread suffering caused by low sexual desire is deployed as a counterargument against the critical view: how insensitive and disrespectful it is to deny treatment to females who suffer. ${ }^{7}$ Sometimes this argument is mixed with suggestions of sexism: the scientific study and therapeutic treatment of sex has for long been androcentric, and now we can help males who suffer from erectile dysfunction, while proponents of the critical view are willing to let females suffer in silence.

Though any form of suffering warrants sympathy, as an argument for the mainstream view the Argument from Suffering is question-begging. It assumes as a premise - that low female sexual desire should be in the domain of medicine - the issue which is under dispute. Not all forms of suffering are in the domain of medicine. One need only consider the suffering caused by hunger or climbing high mountains or listening to country music. Even if we grant that low female sexual desire causes suffering, this does not support the mainstream view on medicalisation of low female sexual desire.

\footnotetext{
${ }^{7}$ Segal (2018) offers a rhetorical analysis of an FDA meeting at which flibanserin was discussed, and she notes that this argument - the suffering caused by an 'unmet medical need' — was one of several offered by promoters of the drug.
} 
Moreover, we will see below that the notion of suffering in this context is contested (§4). Critics hold that the suffering associated with low female sexual desire is typically not an intrinsic harm to the females with the condition, but rather arises as a result of social norms of sexuality or relationship difficulties. To consider an analogy, a homosexual male in present-day Russia might suffer distress from his sexual orientation, not because his sexual orientation is intrinsically harmful (obviously), but because he lives in a society which subordinates and physically harms homosexuals. This rejoinder to the Argument from Suffering is itself inconclusive when deployed against the entire category of low female sexual desire, for reasons we will see in $\S 4$, though it is persuasive for some proportion of cases.

\subsection{The Appetitive Argument}

We saw above that some hold that sexual desire is like an appetite or physiological capacity, and low sexual desire is a result of dysfunction in this capacity. Kinsey, for example, believed that sexual desire is the result of a physiological capacity, akin to the capacity of our pancreas to produce insulin (Kinsey, Pomeroy, and Martin 1948). A low capacity in the latter is a disease (type 1 diabetes), hence a low capacity in the former is also a disease.

A physiological capacity view has been widely adopted by those promoting low female sexual desire as a disease. Some theorise that low female sexual desire is a result of low levels of particular hormones such as testosterone- the Berman sisters are two prominent defenders of the mainstream view who frequently have claimed that low sexual desire in women can be treated with testosterone, and a testosterone patch was being developed for low female sexual desire but was ultimately rejected for consumer use by the FDA (because of concerns about harmful side effects such as heart attacks, breast cancer, and weight gain), though it was approved in Europe. Others theorise that low female sexual desire is a result of an imbalance in neurotransmitters (see for example Croft 2017); this is the basis of the first drug approved for low female sexual desire (flibanserin). After the success of Viagra for erectile dysfunction, its manufacturer began testing it for treating low sexual desire in women. All these attempts to develop pharmaceutical interventions for low female sexual desire assume a physiological capacity view of sexual desire.

One problem with the Appetitive Argument is that it ignores the intentional, psychological, social, and cultural context of sexuality. In §4 I describe some of the substantive ways that this challenge has been articulated, though in $\$ 5 \mathrm{I}$ argue that appealing to the causal aetiology of 
low female sexual desire offers more modest support to the critical view than its defenders suggest. A further problem with the Appetitive Argument is that thus far no physiological basis for low female sexual desire in general has been discerned.

Nevertheless, it is plausible that for some females with low sexual desire, the cause of their low desire is indeed a result of a dysfunction in a physiological capacity. There are reasons to think that some hormone concentrations can influence sexual desire (in both males and females). We have empirical evidence suggesting that modulating physiological states with pharmaceuticals such as selective serotonin reuptake inhibitors can dampen sexual desire, which itself suggests that sexual desire has a biological basis of one form or another (Bala et al. 2018). Though this consideration might have some initial appeal for a defender of the mainstream view, it is in fact far from conclusive. That is because yet another problem with the Appetitive Argument is that many features of life which are non-medical have a grounding in a physiological capacity. Athletic prowess is a good example. One's running speed is a function not only of training but also of an intrinsic physiological capacity. Alexei's slow running speed might be a function of his unusually low intrinsic physiological capacity for running, but that does not entail that Alexei has a disease.

However, the Appetitive Argument together with the Argument from Suffering are jointly persuasive, for at least some cases of low female sexual desire. It is plausible that some cases of low female sexual desire have a physiological aetiology, and that this causes those people to suffer (though in $\$ 4$ we see that this latter premise must be understood with care). There is, thus, some reason to think that at least for some cases of low female sexual desire, those cases are genuine diseases.

\subsection{The Argument from Female Equality}

We saw above that proponents of the mainstream view sometimes frame the medicalisation of female sexual desire as an issue about equality between the sexes. There are grounds for thinking that sex research has been unduly focused on male sexuality. For example, during her research about evolutionary theories of the female orgasm, the philosopher Elizabeth Lloyd traced sociobiologists' footnotes regarding the scientific study of orgasms, and she found that, in the context of theorising about female orgasms, many of the cited sources were in fact based on the study 
of males (see Lloyd 2005; Okruhlik 1994). ${ }^{8}$ With the success of pharmaceutical treatments for erectile dysfunction beginning in the late 1990s, there was an immediate motivation to develop an equivalent intervention for females. The Argument from Female Equality claims that it is only fair that disorders of female desire receive the same attention as their male equivalents. If male sexual dysfunctions can be medicalised, then so can female sexual dysfunctions. This argument was the basis of the name for the recent industry-funded patient advocacy campaign for the drug flibanserin: Even The Score. ${ }^{9}$

This argument has several damning problems. It assumes that low male sexual desire itself ought to be in the domain of medicine. The argument seems to be: if low male sexual desire has been successfully medicalised, then so too should low female sexual desire. But the critical view on the medicalisation of low female sexual desire applies equally to low male sexual desire-critics have argued that male sexuality has been inappropriately medicalised (Tiefer 1986, 1994; Fishman 2007). Moreover, Bueter and Jukola (2020) convincingly argue that feminism has usually been deployed in criticisms of medicalisation and biological reductionism; therefore to cite concerns about female equality as grounds for upholding the disease status of low female sexual desire, with the ultimate aim of warranting pharmaceutical intervention for the condition, is far-fetched.

Sometimes the Argument from Female Equality is made in the context of discussions about interventions. The argument goes: males have access to effective interventions for their sexual dysfunctioning, and therefore so should females. But what, critics have asked, is the female analogy of intervening on erectile dysfunction? One hypothesis that received some study was: just as pharmaceuticals like Viagra work by increasing blood flow to the penis, perhaps some interventions can increase blood flow to the clitoris. A barrier to this approach, however, is that many empirical studies suggest little correlation between physical signs of arousal in females, such as vaginal blood flow, and subjective feelings of arousal and desire. ${ }^{10}$ Similarly, treatment of erectile dysfunction is not in fact an intervention for low male sexual desire, and thus, at least in the context of interventions, the Argument from Female Equality does not bear on whether low female sexual desire should be medicalised.

\footnotetext{
${ }^{8}$ Taylor (2015) and Angel (2012) note the uneasy and complicated relationship between feminism and the medicalisation of low female sexual desire.

${ }^{9}$ See Segal (2018) for a critical account of various articulations of this argument.

${ }^{10}$ Though such findings have been observed for decades, they have been demonstrated in an elegant series of experiments by Meredith Chivers. See Chivers et al. (2010) for a review.
} 


\subsection{The Argument from Treatment Success}

Prominent advocates of the mainstream view have claimed that low female sexual desire can be successfully modulated by pharmaceuticals. This, proponents claim, is a reason to think that low female sexual desire should be in the domain of medicine.

Such proclamations of treatment success are laughable in their hyperbolic contradictions of empirical data. Irwin Goldstein, for instance, claimed that when preparing the FDA submission for flibanserin, the worry was not that the drug would be perceived as enhancing female sexual desire too little, but that it would be perceived as enhancing female sexual desire too much - the company did not want to elicit the concern that the drug would be "turning women into nymphomaniacs". ${ }^{11}$ The drug in question was rejected by the FDA twice, before it was finally approved during the Even The Score campaign. The basis of the rejections were the tiny observed beneficial effects of the drug, and concerns about its harm profile (one trial testing the safety of this drug to treat low sexual desire in females included only males). Earlier attempts to develop testosterone interventions also floundered upon careful evaluation. The second and thus far last drug approved for low female sexual desire (bremelanotide) has an effect size similar to that of flibanserin. On average, compared with placebo, flibanserin is associated with an increase of about one 'sexually satisfying event' every two months (Jaspers et al. 2016).

\section{The Critical View}

Critics have argued that low female sexual desire has been inappropriately medicalised. This charge involves a number of related claims: that low female sexual desire is a normal part of life, that low female sexual desire is not caused by medical problems but rather is caused by social, relational, or cultural factors, that the very idea that low female sexual desire is a problem reflects particular social values, that the best way to help low female sexual desire (assuming help is called for) involves non-medical interventions, and that the condition has been constructed as a disease in part because of the financial gains to be had by selling treatments for it.

The critical view has a range of adherents. The New View Campaign, led by psychologist Leonore Tiefer, is among the more visible organisations

\footnotetext{
${ }^{11}$ In Goldstein's words: "When you're going to the FDA with this kind of drug, there's the sense that you want your effects to be good but not too good (...) there was a lot of discussion about it by the experts in the room, the need to show that you're not turning women into nymphomaniacs. There's a bias, a bias against—a fear of creating the sexually aggressive woman." Cited in Bergner (2014).
} 
defending the critical view, and John Bancroft, the former director of the Kinsey Institute, has also defended the critical view. The journalist Ray Moynihan has published a number of articles and books in which he decries medicalisation practices such as 'disease-mongering' or 'selling sickness', and he has applied such arguments to low female sexual desire. Several academic commentators have aligned themselves with the critical view of medicalising low female sexual desire in scholarly publications (see. e.g. Kaschak and Tiefer 2001; Moynihan 2003; Moynihan and Mintzes 2010; Bancroft 2002; Taylor 2015; Angel 2012; Cacchioni 2015).

The primary arguments for the critical view are:

The Spurious Disease Argument

The Construction of Distress Argument

The Argument from Treatment Failure

The Conflict of Interest Argument

The Harms Argument

I address each in turn, going from subtle to simple.

\subsection{The Spurious Disease Argument}

Sometimes the debate about the medicalisation of a condition involves the claim that the condition is, or is not, a genuine disease. If a condition is a genuine disease, then, goes this thought, it should be in the domain of medicine; if a condition is not a genuine disease, then there is at least some reason to suppose that the condition should not be in the domain of medicine (though medicine does have in its domain conditions that are not diseases, such as pregnancy). In $\S 3$ we saw the Appetitive Argument for the mainstream view. The Spurious Disease Argument for the critical view denies the appetitive model of low sexual desire. Indeed, the charge of medicalisation of low female sexual desire often involves a denial of the capacity view of sexual desire, or at least a denial that the capacity view is a complete explanation for varying strengths of sexual desire. Critics argue that the view of low sexual desire as a deficiency in a physiological capacity is excessively reductionist, and to understand a female's low sexual desire we must take into account that female's broader social context. ${ }^{12}$ To properly understand why a female has low sexual desire, one must consider many features of her life, including her general health, levels

\footnotetext{
${ }^{12}$ See, among many others, Tiefer (1991). Leiblum, for example, claimed that "Inferring that hormones, in general, are the primary motivators of sexual activity in humans is a gross oversimplification" $(2002,65)$.
} 
of stress, competing interests, and features of her past and present relationships.

Taking this contextual approach further, some feminists such as Catherine MacKinnon (1989) argue that a theory of female sexuality must be located within a broader theory of gender inequality. A proper characterisation of female sexual dysfunction should not begin with the assumption that normal healthy human sexual desire is that of males. Male sexual desire is, obviously, itself influenced by social shaping. Moreover, male and female sexual desire is radically different, claims MacKinnon (which is itself a controversial premise). Females who seem to have dysfunctionally low female sexual desire should instead be seen as resisting a male-centric system and standards of sexuality. ${ }^{13}$ Cases of apparent low sexual desireat least many cases - should be understood, argues MacKinnon and others, as appropriate responses to gender inequality and sexual violence.

A more mundane version of the Spurious Disease Argument was voiced by none other than Lori Brotto, a psychologist who chaired the DSM-5 sexuality committee - the group which developed the disease category 'female sexual interest/arousal disorder'. When interviewed about low female sexual desire, Brotto claimed: "Sometimes I wonder whether it isn't so much about libido as it is about boredom". Brotto was referring to the typical decline in sexual desire that occurs in long-term monogamous relationships. ${ }^{14}$

If the Spurious Disease Argument is meant as a thesis about some token instances of low female sexual desire, then it is convincing, since it is surely plausible that for some females diagnosed with the disease, their condition is better understood as arising from their social context rather than from their intrinsic physiological capacities. However, if the Spurious Disease Argument is meant as a thesis about the disease itself, as a kind, then it is less convincing, since the thesis would deny that any particular instance of low female sexual desire could be a case of disease. That, though, would be committed to claiming that there does not exist a female with low sexual desire for whom their condition is a disease. And that is implausible. To see why, consider what any of the leading philosophical theories of disease must say about a female who, for the sake of argument,

\footnotetext{
${ }^{13}$ It is a mistake, argues MacKinnon, to see women with low sexual desire "as in need of explanation and adjustment, stigmatized as inhibited and repressed and asexual" $(1989,141)$

${ }^{14}$ The Brotto interview is reported in (Bergner 2014). During therapy for women diagnosed with low sexual desire, Brotto noted that "the impact of relationship duration is something that comes up constantly". For this reason, Bergner, who conducted this interview, calls drugs like flibanserin less of an intervention for libido and more of an intervention for monogamy.
} 
suffers genuine distress as a result of her low sexual desire (we will see below that this premise requires nuance).

Normativism about disease holds, roughly, that if a condition is disvalued and if medicine can help, then that condition is a disease. For the Spurious Disease Argument to work as a thesis about the disease itself, assuming normativism, one would have to deny either that the condition is disvalued (but we have granted for the sake of argument that the female in question suffers), or that for all females who experience low sexual desire, medicine cannot help. This latter premise is of course empirical, but it is extremely implausible. Naturalism about disease, on the other hand, holds roughly that if a condition involves a statistical departure from normal functioning, and that dysfunctioning impedes with the ultimate aims of survival and reproduction, then that condition is a disease. For the Spurious Disease Argument to work as a thesis about the disease itself, assuming naturalism, one would have to deny that there exists a female whose sexual desire is much lower than the statistical norm and which impedes her survival or reproduction. This, again, is highly implausible. My favoured account of disease is a hybrid account, which also entails that the Spurious Disease Argument cannot be about the disease itself as a general kind. ${ }^{15}$ (It is worth noting that the arguments in this paragraph dodge the question about aetiology altogether - we will return to this in §5.)

To sum: the Spurious Disease Argument may be compelling when understood as thesis about some instances of low female sexual desire, but not when understood about the entire disease category. ${ }^{16}$ Of course, among all the females who are diagnosed with a disease of low sexual desire, the proportion for whom the Spurious Disease Argument applies remains an open question. We have seen several reasons to think that for many females who are diagnosed with a disease of low sexual desire, their condition is better understood in social or cultural terms, and so their diagnosis may be inappropriate. Thus, the Spurious Disease Argument provides less warrant to a general thesis of medicalisation of low female sexual desire, and more warrant to what Gabriel and Goldberg (2014) call 'disease inflation': the expansion of diagnostic categories and the loosening of diagnostic practices and prescription norms such that more and more people are said to be diseased and are prescribed interventions.

\footnotetext{
${ }^{15}$ On normativism, see Cooper (2002). On naturalism, see Boorse (1977). On hybridism, see Stegenga (2015).

${ }^{16}$ Some proponents of the critical view are occasionally slippery on this point. Moynihan, for example, claims that while it is surely true that some females have a genuine disease of low sexual desire, the disease category itself is the "freshest, clearest example" of "the corporate sponsored creation of a disease" (2003).
} 
One further nuance is worth mentioning. The above discussion relied on a distinction between condition types and condition tokens: the Spurious Disease Argument fails as a thesis about the condition type (the general category of low female sexual desire), but it might succeed as a thesis about condition tokens (Sveta's low sexual desire is not a case of genuine disease, it is a result of an abusive marriage). The underlying premise is that claims of medicalisation should apply to condition tokens rather than condition types, because two people could have the same type of condition in which one of the tokens is constituted by a disease and the other is not. But this would only make sense if by 'condition' one meant 'cluster of symptoms': one cluster of symptoms could be caused by a disease, while another cluster of those same symptoms could be caused by some non-disease state (for example, Maria's sadness and crying and sleeplessness are caused by her depression, while Sofia's sadness and crying and sleeplessness are caused by the recent breakup with her spouse). But if by 'condition' one meant 'whole disease entity, including symptoms and physiological causes of those symptoms', then two tokens of a condition would share all physical features, and thus, arguably, two tokens of the same condition would either both be genuine diseases or both be non-disease conditions. All tokens of type 1 diabetes are cases of genuine disease, while all tokens of appreciating country music are non-disease conditions (though distressinducing nevertheless). Since the Spurious Disease Argument fails as a thesis about condition types, it can only succeed as a thesis about some condition tokens. But how could it be, following the above line, that some tokens of a condition are genuine diseases while other tokens of the condition are not genuine diseases, if they are tokens of the same condition? One answer which has tempted many defenders of the critical view, and which we have already touched upon, is to distinguish genuine disease tokens from spurious disease tokens according to the aetiology of those tokens. This, finally, brings us to a remaining nuance for Spurious Disease Argument, which I address in $\$ 5$.

\subsection{The Construction of Distress Argument}

To be diagnosed with female sexual interest/arousal disorder, the DSM stipulates that a female must suffer distress from her symptoms of low sexual desire. At first glance this seems like a reasonable requirement, since the symptoms alone are not necessarily pathological and it is hard to see what other reason medicine could have to hold that a female with such symptoms is diseased. Indeed, many asexuals have no sexual desire at all and yet do not experience distress as a result, and many would deny that they have a disease. However, the requirement that a female experience distress from her symptoms of low desire in order to be diagnosed raises difficult questions. The Construction of Distress argument holds that the 
distress that a female with low sexual desire experiences can be a result of social or cultural features of the female's context, rather than a result of the symptoms themselves (we saw the Construction of Distress Argument foreshadowed as a response to the mainstream position's Argument from Suffering in §3). A female could experience such distress if she felt that she was not satisfying social norms regarding sexual activity or pleasure. Such norms might be generated by manifold social forces, such as peers, advertising, and pornography. Moreover, such norms might be unwarranted or thoroughly pernicious.

The Construction of Distress argument has an additional complexity. Female sexual desire is often deemed low only relative to the strength of their typically male partners. Such distress, in many cases of low female sexual desire, might not be intrinsic, but rather might be relational. That is, such distress can arise not from the female's symptoms directly, but rather from relationship difficulties which arise due to an imbalance of desire with her partner (see, e.g., Irvine 1990). ${ }^{17}$

A curious proviso to the description of female sexual interest/arousal disorder in the DSM-5 notes that there is variability in the prevalence of low sexual desire in different cultures, and cautions:

A judgement about whether low sexual desire reported by a woman from a certain ethnocultural group meets criteria for female sexual interest/arousal disorder must take into account the fact that different cultures may pathologise some behaviors and not others. (APA 2013, 436)

This appears to be a form of cultural relativism regarding whether a case of low female sexual desire should be deemed a disease or not. One might think that this is muddled, since whether a person has a disease should not depend on culture-specific idiosyncrasies regarding whether that culture pathologizes the condition in question. However, such cultural relativism of disease attribution could be reasonable if it is the case that in some cultures a female with low sexual desire experiences distress while in other cultures a female with low sexual desire experiences no distress, due to differences in the extent to which the cultures pathologises low female sexual desire. But this faces the Construction of Distress argument: the distress that females experience because of the pathologizing tendencies of their culture are, trivially, a result of their culture, and not a result of

\footnotetext{
${ }^{17}$ Taylor (2015) notes that many of the alleged cases of successful treatment of low female sexual desire described by the Berman sisters involved females who were distressed as a result of partner frustration (Berman, Berman, and Bumiller 2001).
} 
intrinsic harms caused by the condition itself. The DSM is explicitly asserting that the distress caused by low female sexual desire is a cultural construction-a puzzling gesture of support for the critical view from what could be taken as the bible of the mainstream view.

Responding to the Construction of Distress argument, defenders of the mainstream view claim that the argument ignores or trivialises suffering of some females with low sexual desire (see Jackson 2004). Yet, if the source of the distress is indeed a result of the pathologizing tendency of a society, on its face this suggests that diagnosing the condition as a disease and subsequently treating it with biological interventions is misguided. Further, in §5 I argue that the causal aetiology of complex traits such as strength of sexual desire probably involve causes at multiple scales, including both biological and social causes.

\subsection{The Argument from Treatment Failure}

We have seen that an argument for the mainstream view appeals to claims about the successful treatment of low female sexual desire, and that these claims are empirically implausible. The critical view turns this argument around in the Argument from Treatment Failure, in which the low effectiveness of interventions for low female sexual desire is cited in the context of discussing the condition's medicalisation (see Moynihan 2014). The drugs introduced in the last couple of decades to treat erectile dysfunction are among the most successful pharmacological developments of the last several decades (by various metrics: capacity to modulate the condition, number of prescriptions, number of men taking the drugs, profitability for the manufacturers; but not, obviously, to save lives or mitigate symptoms of mortal diseases). Conversely, only two of many experimental drugs for low female sexual desire have made it through the research and regulatory pipeline, and these drugs have extremely modest beneficial effects for females but significant harms (see below). Drugs to improve low female sexual desire have been failures. One possible explanation for such failures is that the condition is not a genuine disease. The underlying argument is: so far there has been no effective intervention developed for low female sexual desire; if low female sexual desire were a genuine disease, an effective intervention would have, by now, been developed; thus, low female sexual desire is not a genuine disease.

One response to the failure of female desire drugs has been to conclude that female sexuality is complex. Indeed, this appeal to the complexity of female sexual desire formed the basis of criticisms of the development of pharmaceutical interventions for female sexual desire, voiced by academic commentators and feminist advocacy groups, even prior to the empirical 
failures of these drugs. ${ }^{18}$ No wonder such drugs have been failures, goes this argument: male sexual arousal may be physiologically simple, but female sexual desire is not.

Treatment failure can, of course, be merely transient. Our failure to adequately treat type 1 diabetes until Banting and Best's breakthrough did not entail that type 1 diabetes is not a genuine disease. Thus, the Argument from Treatment Failure is far from conclusive for the critical view. Yet, at the very least the Argument from Treatment Failure is a compelling rejoinder to the mainstream view's Argument from Treatment Success.

Moreover, the failure to modulate female desire with pharmaceuticals is not due to a lack of effort on the part of scientists and companies to find such a drug. The fantastic profits to be gained from a female desire drug have spurred an enormous search. This is a case in which absence of evidence is some evidence of absence. ${ }^{19}$ The absence of evidence of effective medical treatments for low female sexual desire is some evidence that there is not going to be an effective medical treatment for low female sexual desire ${ }^{20}$ We have some reason to think, now, that a drug for female sexual desire is not forthcoming. The inability to medically intervene on a condition provides at least some reason for thinking that the condition should not be in the jurisdiction of medicine.

\subsection{The Conflict of Interest Argument}

Sometimes the charge of medicalisation involves describing tactics used by interested parties in convincing others, especially physicians and potential future patients, that a condition is a disease. These tactics include organising meetings of experts with the aim of defining a disease, sponsoring medical education events to inform physicians about the condition, and performing research which suggests that the condition is under-diagnosed and under-treated (Moynihan 2003; Fishman 2004; Cacchioni 2015). The point of these tactics, of course, is to make money by selling interventions for the condition. ${ }^{21}$ Let us call this the Conflict of Interest Argument.

\footnotetext{
${ }^{18}$ See Bueter and Jukola (2020), who argue that the flibanserin case involved a failure in the uptake of criticism, and thus the requirements of Longino's theory of scientific objectivity were not satisfied.

${ }^{19}$ See Sober (2009) for an articulation of the formal conditions under which absence of evidence is indeed evidence of absence, contrary to standard statistical lore.

${ }^{20}$ Hacking's infamous quip "if you can spray them, then they are real" (1983) — originally perhaps an unintended innuendo but here an unapologetic pun - might be apt here.

${ }^{21}$ As Taylor puts it: "The diagnosis is not about illness or abnormality; it is about making large numbers of people think that they are ill or abnormal so that corporations can profit" (2015).
} 
With respect to the question of medicalisation, an implicit premise of this argument seems to be that such tactics would be unnecessary if the condition were in fact a real disease. However, the same tactics cited in the argument - corporate-funded consensus conferences, medical education, awareness-raising campaigns, patient-advocacy groups-are deployed against genuine diseases, such as breast cancer, HIV, and depression. The Conflict of Interest Argument has some rhetorical sway, but is ultimately inconclusive as a consideration pertinent to medicalisation. That is not to say that conflicts of interest are not an important problem in medicine, in medical research, or in debates about the medical status of some conditions. Holman and Geisler (2018) use the case of flibanserin to show that in FDA consultation meetings, financial conflicts of interest appeared to influence the content of testimony offered by patient advocacy panelists, which in turn probably influenced the FDA decision to approve the drug (see also Segal 2018). Conflicts of interest almost surely had some causal influence on the determination of the putative disease status of low female sexual desire. Yet the same kinds of conflicts of interest are present in many areas of medicine and themselves do not necessarily impugn the medical status of a condition.

\subsection{The Harms Argument}

The potential harms of the medicalisation of low female sexual desire are numerous. The Harms Argument just says: the potential harms of medicalising low sexual desire are reasons not to medicalise the condition. One class of harms is the various adverse effects of the medical interventions used to treat low female sexual desire. At present this is primarily the drug flibanserin, which has several harmful effects, including fatigue, insomnia, and hypotension. ${ }^{22}$ Another kind of harm is the reification of spurious and pernicious norms of sexuality. ${ }^{23}$ Reiheld argues that in general medicalisation can have the harm of reification, defined as "a process whereby the ontology of an idea shifts from mere concept to real manifestation" $(2010,77)$. One way this might occur is via looping effects of human classification, in which those people who are diagnosed with a condition come to see themselves and be seen and treated by others

\footnotetext{
${ }^{22}$ Taylor argues that "the medical treatment of FSD, as with the medical management of menopause, subjects women to health risks and disciplinary treatments in order to accommodate men and to maintain heterosexual marriages" $(2015,43)$.

${ }^{23}$ As John Bancroft, former director of the Kinsey Institute, claimed "The danger of portraying sexual difficulties as a dysfunction is that it is likely to encourage doctors to prescribe drugs to change sexual function - when the attention should be paid to other aspects of the woman's life. It's also likely to make women think they have a malfunction when they do not." (Quoted in Moynihan 2003). Wardrope (2015) argues that critiques of medicalisation can involve claiming that medicalisation involves 'hermeneutical injustice'. See also de Vries (2007), Verweij (1999), and Gagné-Julien (2021 this issue of EuJAP).
} 
as fundamentally a kind of person (the kind with that condition), and thereby in various ways they become that kind of person. ${ }^{24}$ Medicalising any condition entails a range of financial costs. Finally, attention can be drawn away from the important causes of low female sexual desire.

While these are important consideration, the Harms Argument is far from conclusive, since the medicalisation of all conditions comes with harm. Moreover, as Reiheld (2010) argues, medicalisation can also have benefits that offset or outweigh such harms, such as the demarginalisation of previously marginalised patient groups and destigmatisation of previously stigmatised conditions. Yet, at least in the case of low female sexual desire, and considering the Argument from Treatment Failure, the two arguments suggest that the benefit-harm ratio for medicalising low female sexual desire is poor. I argue in the following section that this pragmatic concern is among the most persuasive, albeit simplest, of the arguments for the critical view.

\section{Etiological Models of Low Desire}

Thus far we have seen several theories about the aetiology of low female sexual desire. One main family of etiological models is based on physiological capacity for sexual desire, and the other main family of etiological modes is based on social context relevant to sexual desire. Proponents of the mainstream view have tended toward the physiological capacity models, whereas proponents of the critical view have tended toward the social context models.

The physiological family of models states that people's capacity for sexual desire varies, and low sexual desire is simply the result of underlying physiological causes, such as low testosterone levels or an imbalance in neurotransmitters. We saw above that this kind of model was favoured by Kinsey, and it is widely held today by pharmaceutical companies. A version of a social context etiological model for sexuality is the repression model, famously articulated by Freud, which states that people's sexual desires are psychogenic, and can be modulated (mildly or extremely, leading in some cases to paraphilias) by psychological mechanisms. Another version of a social context etiological model is the oppression model, which states that females' sexual desires are modulated by gender inequality, stress, fatigue, and fear of violence. This has been defended by feminists such as MacKinnon. Still another version of a social context etiological model is the boredom model, which states that the strength of

${ }^{24}$ This is Hacking's (1995) "looping effects of human kinds". 
sexual desire wanes in particular contexts, especially as a result of relationship duration.

These models are not mutually exclusive, of course - low sexual desire can have multiple aetiologies. However, some of the more prominent defenders of the various models have tended to emphasise one model at the expense of the others. Kinsey, for example, downplayed the importance of social context as an explanation for low sexual desire and emphasised physiological capacity. ${ }^{25}$ MacKinnon, conversely, downplayed the importance of physiological capacity and emphasised social context. Yet, all these aetiological models have some initial plausibility.

We saw above that appealing to the aetiology of token instances of low female sexual desire could be a way to distinguish cases of low sexual desire which should be understood as genuine diseases from cases of low sexual desire which should not be understood as genuine diseases. The underlying premise of some appeals to the social context etiological models is that if a female's low sexual desire is due to social or cultural causes, then this female does not have a disease, and thus to diagnose her with a disease amounts to inappropriately medicalising her condition.

As persuasive as this claim may be, this line of argumentation requires care to avoid an ambiguity regarding causation of disease.

Many conditions that people consider to be uncontroversially in the domain of medicine arise from causes that are, ultimately, social or cultural. Car accidents, sporting injuries, drug overdoses, and nuclear reactor meltdowns can all lead to conditions that are medical. In a trivial sense these causes of conditions are all social or cultural artefacts, yet we would not say that the resulting conditions are not genuine diseases. Wellstocked grocery stores and liquor stores and pharmacies are the causes of a wide range of diseases, almost surely more than diseases caused by intrinsic physiological dysfunctions. A person's social context can cause a wide range of genuine diseases.

The distinction between social or cultural causes on the one hand and physiological causes on the other is less sharp than one might suppose. We have some understanding of the pathophysiological mechanisms in which infection with Mycobacterium tuberculosis causes symptoms of tuberculosis. But we also have some understanding of the mechanisms in

\footnotetext{
${ }^{25}$ Kinsey "consistently ignored the ways in which women as a social group may have been taught to avoid or dislike sex and sought biological explanations for their supposedly lower sexual capacity" (Irvine 1990, 40).
} 
which the social context of a prisoner in a crowded jail in Kyrgyzstan causes infection with, and subsequent symptoms of, tuberculosis. ${ }^{26}$ It is plausible that for many human conditions such as the strength of one's sexual desire, the etiological causal nexus is extremely complex, and the relevant causes exist at various physical scales, from the chemical to the social, and various temporal scales, from the temporally distal to the temporally proximal.

Perhaps what defenders of the critical view have in mind when they appeal to social or cultural models of aetiology of low female sexual desire is a distinction between proximal causes of a disease and distal causes of a disease. The presence of Mycobacterium tuberculosis is a proximal cause of symptoms. But how did the prisoner get infected with this bacterium? To explain this adequately one must cite the distal, social cause: jail overcrowding. This is a small victory for the critical view on medicalisation of low female sexual desire, however, because if our interest is in whether a condition is a genuine disease, then all that matters in our hypothetical case is the proximal cause, namely, the presence of the infectious bacterium. Since infectious diseases are far less controversially held to be genuine diseases, we have an argument that diagnosis by appeal to proximal causes of symptoms, and not distal causes, is not merely sanctioned by medical practice but is in fact normal medical practice. Why should diseases of sexual desire be any different?

To give a concrete example of this in the debate about the medicalisation of low female sexual desire, in an insightful article about the medicalisation of female sexual dysfunction (FSD), Taylor argued that "Much of the problem with FSD seems to arise from lack of education, rather than from something aberrant about the women" $(2015,263)$. While this is almost certainly true, it is also true for many conditions that are uncontroversial diseases. When Alexei tells Mischa that it is safe to ski on this black diamond ski slope, or that he should take the blue pill rather than the red pill, or that one drives on the left side of the road in Canada, Mischa's resulting dysfunctions arise from a lack of education (both his and Alexei's), rather than anything aberrant about Mischa. And yet those dysfunctions could be genuine diseases.

There is an important analogy with recent debates about depression, and because the pertinent arguments are similar, it is worth considering them. In the DSM-IV, the diagnostic category for depression had a 'bereavement exclusion criterion', such that a person who satisfied the symptomatic

\footnotetext{
${ }^{26}$ Furman (2017) applies such reasoning to argue that a full understanding of AIDS requires both physiological and social models.
} 
criteria for depression was excluded from a diagnosis of depression if they were bereaving. The thought was that a bereaving person's symptoms of depression are better explained by the fact that they have lost a loved one rather than by the hypothesis that they have a disease (Horwitz and Wakefield 2007). Thus the bereavement exclusion criterion amounted to a consideration of a person's social context when determining if that person has a disease (though the social context that was considered was narrow: there was no 'recently unemployed exclusion criterion' or 'listened to excessive Nick Cave albums exclusion criterion' or 'broke up with girlfriend exclusion criterion'). Some commentators noted that bereavement does not immunise one against depression, and indeed, the loss of a loved one can cause depression - not just apparent symptoms of depression, but depression itself. So when revising the description of the disease category for the next edition of the DSM (DSM-5), the bereavement exclusion criterion was eliminated. Critics who had argued that the bereavement exclusion criterion did not go far enough in considering people's social context were disappointed. However, we have seen that this appeal to social context in determining the status of a condition as a disease is inconclusive.

In the DSM-5, the diagnostic criteria for female sexual interest/arousal disorder also stipulates a diagnostic exclusion criterion, based on social context. It reads as follows:

If interpersonal or significant contextual factors, such as severe relationship distress, intimate partner violence, or other significant stressors, explain the sexual interest/arousal symptoms, then a diagnosis of female sexual interest/arousal disorder would not be made. (APA 2013, 436)

Here the DSM makes a significant nod to social context aetiological models of low female sexual desire. But just as with depression, the deployment of such exclusion criteria assumes that there is a sharp distinction between social causes and physiological causes of a disease, which, I argued above, is not generally true. Presumably the "significant stressors" referred to in the exclusion criterion could itself cause disease, including low female sexual desire. Perhaps what the APA has in mind is that among cases of low female sexual desire, those cases with clear socialcontext aetiologies should not be deemed cases of disease, while other cases should be; perhaps the assumption is that the remaining cases have a physiological aetiology. But why assume that the latter have a physiological aetiology? More pressing, why assume that the former do not have a physiological aetiology? We have seen that many conditions can have a social-context aetiology and be characterised by underlying 
physiological states. Perhaps the APA (and proponents of the critical view) believes that low sexual desire is not one of those kinds of conditions. In any case, this exclusion criterion amounts to holding that one set of possible causes of low sexual desire (biological) should be de-emphasised when another set of possible causes (social) is present.

The most plausible way of making sense of this social-context exclusion criterion for diagnosing low female sexual desire is pragmatic. The exclusion criterion makes sense in the context in which medical interventions can do little good for low female sexual desire in general, while the various factors stipulated as excluding a diagnosis-severe relationship distress, partner violence, or other stressors - can, at least in some cases (one optimistically hopes), be modified, and thus targeting social causes of low sexual desire can do much more good than targeting alleged physiological causes. Flibanserin may not help many females' low sexual desire, but ending an abusive relationship might. Moreover, in addition to the known adverse effects that medications for low female sexual desire have on the body, one might worry about another sort of indirect harm: low desire which is a result of a female's social context (relationship problems or work stress or ...) might be a cue to modify this context (modify or end the bad relationship, for example), and medicating away that low desire (assuming that such interventions were in fact effective at increasing sexual desire) could silence this cue, and thus decrease the motive for positive change.

Contrast this with erectile disorder. The DSM description for erectile disorder stipulates a similar exclusion criterion (the symptoms must not be better explained by relationship distress or other stressors). Now imagine Sergei, who is in a distressing relationship and has begun to experience symptoms of erectile dysfunction. His rule-following physician is forbidden from making a diagnosis of erectile disorder, despite the fact that she knows that an effective intervention is available. While it might be prudent for Sergei to reconsider aspects of his relationship, it would be excessively prudish to deny him the effective treatment that is now available, on the grounds that his condition has a social-context etiology. ${ }^{27}$ This is not to say that the social-context etiological model is not important for Sergei; the same concern about an unintended mitigation of the motive for positive change applies. My suggestion here is pragmatic: since we have effective and relatively safe interventions for erectile disorder, worrying about whether Sergei has a genuine disease is fussy.

\footnotetext{
${ }^{27}$ Which might explain why in some jurisdictions, such as the United Kingdom, one can purchase Viagra without a prescription or diagnosis.
} 
This pragmatic consideration-which foregrounds the consequences of deeming a condition a disease and asks whether medicine can effectively intervene on the condition - can inform a general approach to debates about the medicalisation of particular conditions. This approach sidesteps the need to determine whether a condition is a genuine disease according to a general philosophical theory of disease. This pragmatic approach is perhaps what lies at the heart of the critical view of the medicalisation of low female sexual desire, since interventions for low female sexual desire have been essentially failures, and, as the critical view notes, such medicalisation runs the risk of mitigating motivation for changing one's social context. The concern about mitigating one's motive for positive change suggests that there is an ethical dimension to this pragmatic consideration. Both the pragmatic and ethical considerations are about the consequences of intervening on low female sexual desire, rather than whether low female sexual desire as a condition is or is not a genuine disease.

\section{Conclusion}

In my survey of some of the primary arguments for the mainstream view, which holds that low female sexual desire should be under medical jurisdiction, I found most of the arguments on both sides inconclusive. All the arguments for the mainstream view are problematic, which itself lends some support to the critical view, since the status quo has little warrant (§3). However, the Argument from Suffering together with the Appetitive Argument lends some support to the conclusion that at least some cases of low female sexual desire belong in the domain of medicine.

The arguments for the critical view, however, are on somewhat firmer ground (§4). The Construction of Distress Argument, while perhaps not applying to all females with low sexual desire, presumably applies to many. However, both the Spurious Disease Argument and the Construction of Distress Argument involve appeals to social context etiological models of low sexual desire, which, I argued in $\$ 5$, is less convincing than proponents of the critical view claim.

The most persuasive arguments for the critical view, I argued, involve pragmatic considerations of the harms and benefits of interventions for low female sexual desire. We have good reasons to think that medicine can do little for females with low sexual desire, and we also have good reasons to think that medicalising female sexual desire causes harms, and these considerations, while simpler than the various inconclusive arguments regarding the genuine disease status of low female sexual desire, are 
enough to doubt whether low female sexual desire ought to be in the domain of medicine.

\section{Acknowledgments}

I am grateful to Anke Bueter, Erin Nash, Saana Jukola, two anonymous referees, and audiences at several conferences and universities for discussion and commentary.

\section{REFERENCES}

American Psychiatric Association. 1952. The Diagnostic and Statistical Manual of Mental Disorders. Washington, DC: American Psychiatric Association.

American Psychiatric Association. 1968. DSM-II: Diagnostic and Statistical Manual of Mental Disorders, 2nd edition. Washington, DC: American Psychiatric Association.

American Psychiatric Association. 1980. DSM-III: Diagnostic and Statistical Manual of Mental Disorders, 3rd edition. Washington, DC: American Psychiatric Association.

American Psychiatric Association. 1987. DSM-III-R: Diagnostic and Statistical Manual of Mental Disorders, 3rd edition, revised. Washington, DC: American Psychiatric Association.

American Psychiatric Association. 1994. DSM-IV: Diagnostic and Statistical Manual of Mental Disorders, 4th edition. Washington, DC: American Psychiatric Association.

American Psychiatric Association. 2000. DSM-IV-TR: Diagnostic and Statistical Manual of Mental Disorders, 4th edition, text revision. Washington, DC: American Psychiatric Association.

American Psychiatric Association. 2013. DSM-5: Diagnostic and Statistical Manual of Mental Disorders, 5th edition. Washington, DC: American Psychiatric Association.

Angel, Katherine. 2010. "The History of 'Female Sexual Dysfunction' as a Mental Disorder in the 20th Century." Current Opinion in Psychiatry 23 (6): 536-41.

https://doi.org/10.1097/YCO.0b013e32833db7a1.

2012. "Contested Psychiatric Ontology and Feminist Critique:

'Female Sexual Dysfunction' and the Diagnostic and Statistical Manual." History of the Human Sciences 25 (4): 3-24. https://doi.org/10.1177/0952695112456949.

Bachmann, Gloria. 2006. "Female Sexuality and Sexual Dysfunction: Are We Stuck on the Learning Curve?" The Journal of Sexual 
Medicine 3 (4): 639-45. https://doi.org/10.1111/j.17436109.2006.00265.x.

Bancroft, John. 2002. "The Medicalization of Female Sexual Dysfunction: The Need for Caution." Archives of Sexual Behavior 31 (5): 45155. https://doi.org/10.1023/A:1019800426980.

Basson, Rosemary. 2000. "The Female Sexual Response: A Different Model." Journal of Sex \& Marital Therapy 26 (1): 51-65. https://doi.org/10.1080/009262300278641.

Bergner, Daniel. 2014. What Do Women Want?: Adventures in the Science of Female Desire. CCCO: an imprint of CollinsHarpers Publishers

Berman, Jennifer, Laura Berman, and Elisabeth Bumiller. 2001. For Women Only: A Revolutionary Guide to Reclaiming Your Sex Life. New York: Henry Holt and Co.

Berman, Jennifer R, Laura Berman, and Irwin Goldstein. 1999. "Female Sexual Dysfunction: Incidence, Pathophysiology, Evaluation, and Treatment Options.” Urology 54 (3): 385-91.

https://doi.org/10.1016/S0090-4295(99)00230-7.

Boorse, Christopher. 1977. "Health as a Theoretical Concept." Philosophy of Science 44 (4): 542-73. https://doi.org/10.1086/288768.

Brotto, Lori A. 2010. "The DSM Diagnostic Criteria for Hypoactive Sexual Desire Disorder in Women." Archives of Sexual Behavior 39 (2): 221-39. https://doi.org/10.1007/s10508-009-9543-1.

Bueter, Anke, and Saana Jukola. 2020. "Sex, Drugs, and How to Deal with Criticism: The Case of Flibanserin." In Uncertainty in Pharmacology, edited by Adam LaCaze and Barbara Osimani, 338:451-70. Boston Studies in the Philosophy and History of Science. Cham: Springer International Publishing. https://doi.org/10.1007/978-3-030-29179-2_20.

Cacchioni, Thea. 2015. Big Pharma, Women, and the Labour of Love. University of Toronto Press. https://doi.org/10.3138/9781442694101.

Chivers, Meredith L., Michael C. Seto, Martin L. Lalumière, Ellen Laan, and Teresa Grimbos. 2010. "Agreement of Self-Reported and Genital Measures of Sexual Arousal in Men and Women: A Meta-Analysis." Archives of Sexual Behavior 39 (1): 5-56. https://doi.org/10.1007/s10508-009-9556-9.

Cooper, Rachel. 2002. "Disease." Studies in History and Philosophy of Science Part C: Studies in History and Philosophy of Biological and Biomedical Sciences 33 (2): 263-82.

https://doi.org/10.1016/S0039-3681(02)00018-3.

Croft, Harry A. 2017. "Understanding the Role of Serotonin in Female Hypoactive Sexual Desire Disorder and Treatment Options." The Journal of Sexual Medicine 14 (12): 1575-84. 
https://doi.org/10.1016/j.jsxm.2017.10.068.

Fishman, Jennifer. 2007. Making Viagra: From Impotence to Erectile Dysfunction. New York: Medicating Modern America: Prescription Drugs in History. A. Tone and E. S. Watkins. New York University Press.

Fishman, Jennifer R. 2004. "Manufacturing Desire: The Commodification of Female Sexual Dysfunction.” Social Studies of Science 34 (2): 187-218. https://doi.org/10.1177/0306312704043028.

Foucault, Michel. 1979. The History of Sexuality, Volume 1: An Introduction. Allen Lane.

Freud, Sigmund. 1905. Three Essays on the Theory of Sexuality. Standard Edition of the Complete Psychological Works of Sigmund Freud. Trans. James Strachey. Basic Books. https://books.google.hr/books?id=AUvjxQEACAAJ.

Furman, Katherine. 2020. "Mono-Causal and Multi-Causal Theories of Disease: How to Think Virally and Socially about the Aetiology of AIDS." Journal of Medical Humanities 41 (2): 107-21. https://doi.org/10.1007/s10912-017-9441-9.

Gabriel, Joseph M., and Daniel S. Goldberg. 2014. "Big Pharma and the Problem of Disease Inflation." International Journal of Health Services 44 (2): 307-22. https://doi.org/10.2190/HS.44.2.h.

Gagné-Julien, Anne-Marie. 2021. "Wrongful Medicalization and Epistemic Injustice in Psychiatry: The Case of Premenstrual Dysphoric Disorder." European Journal of Analytic Philosophy 17. https://doi.org/10.31820/ejap.17.3.3.

Hacking, Ian. 1983. Representing and Intervening: Introductory Topics in the Philosophy of Natural Science. Cambridge, UK: Cambridge University Press.

1995. "The Looping Effects of Human Kinds." In Causal Cognition, edited by Dan Sperber, David Premack, and Ann James Premack, 351-83. Oxford University Press. https://doi.org/10.1093/acprof:oso/9780198524021.003.0012.

Holman, Bennett, and Sally Geislar. 2018. "Sex Drugs and Corporate Ventriloquism: How to Evaluate Science Policies Intended to Manage Industry-Funded Bias." Philosophy of Science 85 (5): 869-81. https://doi.org/10.1086/699713.

Horwitz, Allan V., and Jerome C. Wakefield. 2007. The Loss of Sadness: How Psychiatry Transformed Normal Sorrow into Depressive Disorder. Oxford: Oxford University Press.

Irvine, Janice M. 1990. Disorders of Desire: Sexuality and Gender in Modern American Sexology. Rev. and Expanded ed. Philadelphia, Pa: Temple University Press.

Jaspers, Loes, Frederik Feys, Wichor M. Bramer, Oscar H. Franco, Peter Leusink, and Ellen T. M. Laan. 2016. "Efficacy and Safety of 
Flibanserin for the Treatment of Hypoactive Sexual Desire Disorder in Women: A Systematic Review and Meta-Analysis." JAMA Internal Medicine 176 (4): 453.

https://doi.org/10.1001/jamainternmed.2015.8565.

Kaschak, Ellyn, and Leonore Tiefer. 2001. A New View of Women's Sexual Problems. New York; London: The Haworth Press. http://www.vlebooks.com/vleweb/product/openreader?id=none \&isbn $=9781317788140$.

Kinsey, Alfred Charles, Wardell Baxter Pomeroy, and Clyde Eugene Martin. 1948. Sexual Behavior in the Human Male. 15. printing. Philadelphia: Saunders Company.

Kleinplatz, Peggy J. 2018. "History of the Treatment of Female Sexual Dysfunction(s)." Annual Review of Clinical Psychology 14 (1): 29-54. https://doi.org/10.1146/annurev-clinpsy-050817-084802. Lloyd, Elisabeth Anne. 2005. The Case of the Female Orgasm: Bias in the Science of Evolution. Cambridge, Massachusetts: Harvard University Press.

MacKinnon, Catharine A. 1991. Toward a Feminist Theory of the State. Cambridge, Massachusetts: Harvard University Press.

Masters, William, and Virginia Johnson. 1966. Human Sexual Response. Boston: Little, Brown and Company.

Meana, Marta. 2010. "Elucidating Women's (Hetero)Sexual Desire: Definitional Challenges and Content Expansion." Journal of Sex Research 47 (2-3): 104-22. https://doi.org/10.1080/00224490903402546.

Moynihan, R. 2003. "The Making of a Disease: Female Sexual Dysfunction." BMJ 326 (7379): 45-47.

https://doi.org/10.1136/bmj.326.7379.45.

Moynihan, Ray. 2014. "Evening the Score on Sex Drugs: Feminist Movement or Marketing Masquerade?" BMJ 349 (oct17 8): g6246-g6246. https://doi.org/10.1136/bmj.g6246.

Moynihan, Ray, and Barbara Mintzes. 2010. Sex, Lies, and Pharmaceuticals: How Drug Companies Plan to Profit from Female Sexual Dysfunction. Greystone Books.

Okruhlik, Kathleen. 1994. "Gender and the Biological Sciences." Canadian Journal of Philosophy Supplementary Volume 20: 2142. https://doi.org/10.1080/00455091.1994.10717393.

Reiheld, Alison. 2010. "Patient Complains of ...: How Medicalization Mediates Power and Justice." IJFAB: International Journal of Feminist Approaches to Bioethics 3 (1): 72-98. https://doi.org/10.3138/ijfab.3.1.72.

Segal, Judy Z. 2018. "Sex, Drugs, and Rhetoric: The Case of Flibanserin for 'Female Sexual Dysfunction." Social Studies of Science 48 (4): 459-82. https://doi.org/10.1177/0306312718778802. 
Simes, Stephen M., and Michael C. Snabes. 2011. "Reaction to the Recent Publication by Rosemary Basson Entitled 'Testosterone Therapy for Reduced Libido in Women."' Therapeutic Advances in Endocrinology and Metabolism 2 (2): 95-96. https://doi.org/10.1177/2042018811404034.

Stegenga, Jacob. 2015. "Effectiveness of Medical Interventions." Studies in History and Philosophy of Science Part C: Studies in History and Philosophy of Biological and Biomedical Sciences 54: 3444. https://doi.org/10.1016/j.shpsc.2015.06.005.

Taylor. 2015. "Female Sexual Dysfunction, Feminist Sexology, and the Psychiatry of the Normal." Feminist Studies 41 (2): 259. https://doi.org/10.15767/feministstudies.41.2.259.

Tiefer, Leonore. 1986. "In Pursuit of the Perfect Penis: The Medicalization of Male Sexuality." American Behavioral Scientist 29 (5): 579 99. https://doi.org/10.1177/000276486029005006.

1994. "The Medicalization of Impotence: Normalizing Phallocentrism." Gender \& Society 8 (3): 363-77. https://doi.org/10.1177/089124394008003005.

Verweij, Marcel. 1999. "Medicalization as a Moral Problem for Preventive Medicine." Bioethics 13 (2): 89-113. https://doi.org/10.1111/1467-8519.00135.

Vries, Jantina de. 2007. "The Obesity Epidemic: Medical and Ethical Considerations." Science and Engineering Ethics 13 (1): 55-67. https://doi.org/10.1007/s11948-007-9002-0.

Wardrope, Alistair. 2015. "Medicalization and Epistemic Injustice." Medicine, Health Care and Philosophy 18 (3): 341-52. https://doi.org/10.1007/s11019-014-9608-3.

Weinrich, James D. 2014. "Notes on the Kinsey Scale." Journal of Bisexuality 14 (3-4): 333-40. https://doi.org/10.1080/15299716.2014.951139. 